\title{
UNSTEADY FREE COVECTION OSCILLATORY COUETTE FLOW THROUGH A POROUS MEDIUM WITH PERIODIC WALL TEMPERATURE
}

\author{
PAWAN KUMAR SHARMA, BHUPENDRA KUMAR SHARMA \\ AND R. C. CHAUDHARY
}

\begin{abstract}
This communication investigates the effect of free convection on the heat transfer and the flow through a highly porous medium bounded by two vertical parallel porous plates. It is assume that free stream velocity oscillates in times about a constant mean. Assuming periodic temperature at the moving plate, the approximate solutions for velocity field, temperature field, skin-friction and the rate of heat transfer are obtained and discussed with the help of graphs and tables.
\end{abstract}

\section{Introduction}

The phenomenon of free convection arises in the fluid when temperature changes cause density variation leading to buoyancy forces acting on the fluid elements. This can be seen in our everyday life in the atmospheric flow, which is driven by temperature differences. Now, free convective flow past a vertical plate has been studied extensively by Ostrach 1, 2] and many others. These studies are confined to steady flows only. In case of unsteady free convective flows, Soundalgekar [3] studied the effects of viscous dissipation on the flow past an infinite vertical porous plate. It was assumed that the plate temperature oscillates in such a way that its amplitude is small. Flows of fluid through porous media are of principal interest because these are quite prevalent in nature. Such flows have attracted the attention of a number of scholars due to their applications in many branches of science and technology. Raptis [4] studied the unsteady free convection flow through a porous medium. Raptis and Peridikis 5 f further studied the unsteady free convection flow through a highly porous medium bounded by an infinite porous plate. The convection in a porous medium with inclined temperature gradient have been studied by Nield [6]. Kelleher et al. 7] also studied the heat transfer response of laminar free convection boundary layers along vertical heated plates to surface-temperature oscillations. The laminar free convection from a vertical plate has been investigated by Martynenko et al. 8]. Harris et al. 9] studied free convection from a vertical plate through a porous media. Das et al. 10] have been studied the transient free convection flow past an infinite

Received April 7, 2004; revised June 14, 2006.

Key words and phrases. Porous medium, couette flow, heat transfer. 
vertical plate with periodic temperature variation. Free convective flow through a porous medium between two vertical parallel plates has been studied by Singh 11]. The object of the present paper is to investigate the effects of free convection on oscillatory Couette flow through a highly porous medium when the temperature of the plate oscillates in time.

\section{Formulation of the problem}

We consider the unsteady Couette flow of a viscous incompressible fluid through a highly porous medium bounded between two infinite vertical porous plates. One of which is suddenly moved from rest with a free stream velocity that oscillate in time about a constant mean. Further, it is assumed that the temperature of moving plate fluctuates in time about a non-zero constant mean. We take $x^{*}$-axis along the moving vertical plate in the vertically upward direction and $y^{*}$-axis is taken normal to this plate. The other stationary vertical plate is assumed to be situated at $y^{*}=b$ at temperature $T_{s}^{*}$. We consider the free-stream velocity distribution of the form:

$$
U^{*}\left(t^{*}\right)=U_{0}\left(1+\varepsilon e^{i \omega^{*} t^{*}}\right),
$$

where $U_{0}$ is the mean constant free-stream velocity, $\omega^{*}$ is the frequency of oscillations and $t^{*}$ is the time. The equations governing the problem are:

Momentum equation

$$
\rho \frac{\partial u^{*}}{\partial t^{*}}=-\frac{\partial P}{\partial x^{*}}+\mu \frac{\partial^{2} u^{*}}{\partial y^{* 2}}-\rho g-\frac{u^{*} \mu}{k^{*}} .
$$

Energy equation

$$
\frac{\partial T^{*}}{\partial t^{*}}=\alpha \frac{\partial^{2} T^{*}}{\partial y^{* 2}}
$$

Boundary conditions

$$
\left.\begin{array}{l}
y^{*}=0: u^{*}=U_{0}\left(1+\varepsilon e^{1 \omega^{*} t^{*}}\right), T^{*}=T_{n}^{*}+\varepsilon\left(T_{n}^{*}-T_{s}^{*}\right) e^{i \omega^{*} t^{*}}, \\
y^{*}=b: u^{*}=0, T^{*}=T_{s}^{*} .
\end{array}\right\}
$$

where $u^{*}, U^{*}, \rho, \mu, P, g, \beta, k^{*}, \alpha, T^{*}, T_{n}^{*}, T_{s}^{*}$ are respectively, velocity, free-stream velocity, density, viscosity, pressure, garvity, volumetric coefficient of thermal expansion, permeability parameter, thermal diffusivity, temperature of fluid in the boundary layer, temperature of the moving plate and temperature of the stationary plate. The $(*)$ stands for dimensional quantities.

Equation (2), for the free stream, is reduced to

$$
\rho \frac{d U^{*}}{d t^{*}}=-\frac{\partial P}{\partial x^{*}}-g \rho_{\infty}-\frac{U^{*} \mu}{k^{*}} .
$$

From equations (2) and (5), we get

$$
\rho \frac{\partial u^{*}}{\partial t^{*}}=\rho \frac{d U^{*}}{d t^{*}}+\mu \frac{\partial^{2} u^{*}}{\partial y^{* 2}}+g\left(\rho_{\infty}-\rho\right)-\frac{\left(u^{*}-U^{*}\right) \mu}{k^{*}} .
$$


This equation is reduced to

$$
\rho \frac{\partial u^{*}}{\partial t^{*}}=\rho \frac{d U^{*}}{d t^{*}}+\mu \frac{\partial^{2} u^{*}}{\partial y^{* 2}}+\rho g \beta\left(T^{*}-T_{s}^{*}\right)-\frac{\left(u^{*}-U^{*}\right) \mu}{k^{*}} .
$$

by using the constitutive equation

$$
g\left(\rho_{\infty}^{*}-\rho^{*}\right)=g \beta \rho\left(T^{*}-T_{s}^{*}\right),
$$

where $\beta$ is the volumetric coefficient of thermal expansion and $\rho_{\infty}$ the density of the fluid far away the surface.

Introducing the following non-dimensional quantities

$$
\begin{aligned}
& y=y^{*} / b, \quad u=u^{*} / U_{0}, \quad U=U^{*} / U_{0}, \quad t=\omega^{*} t^{*}, \quad \omega=\omega^{*} b^{2} / \nu, \\
& \theta=\left(T^{*}-T_{s}^{*}\right) /\left(T_{n}^{*}-T_{s}^{*}\right), \quad G r(\text { Grassoff number })=\frac{g \beta b^{2}\left(T_{n}^{*}-T_{s}^{*}\right) \theta}{\nu U_{0}}, \\
& k=k^{*} / b^{2}, \quad \operatorname{Pr}(\text { Prandtl number })=\nu / \alpha .
\end{aligned}
$$

The equations (6) and (3) become

$$
\begin{aligned}
& \omega \frac{\partial u}{\partial t}=\omega \frac{\partial U}{\partial t}+\frac{\partial^{2} u}{\partial y^{2}}+G r \theta-\frac{(u-U)}{k}, \\
& \omega \operatorname{Pr} \frac{\partial \theta}{\partial t}=\frac{\partial^{2} \theta}{\partial y^{2}}
\end{aligned}
$$

with corresponding boundary conditions

$$
\left.\begin{array}{ll}
y=0: & u=1+\varepsilon e^{i t}, \theta=1+\varepsilon e^{i t}, \\
y=1: & u=0, \theta=0 .
\end{array}\right\} .
$$

\section{Solution of the problem}

Since the amplitudes of the free-stream velocity and temperature variation $\varepsilon(\ll 1)$ is very small, we now assume the solutions of the following form:

$$
\left.\begin{array}{l}
u(y, t)=u_{0}(y)+\varepsilon u_{1}(y) e^{i t}, \\
\theta(y, t)=\theta_{0}(y)+\varepsilon \theta_{1}(y) e^{i t},
\end{array}\right\}
$$

and for the free-stream velocity

$$
U=1+\varepsilon e^{i t} .
$$

Substituting equations (10) and (11) in equations (7) and (8), comparing the coefficients of identical power of $\varepsilon$ and neglecting those of $\varepsilon^{2}$, we get following equations

$$
\begin{aligned}
& u_{0}^{\prime \prime}-\frac{u_{0}}{k}=-G r \theta_{0}-\frac{1}{k}, \\
& \theta_{0}^{\prime \prime}=0 \\
& u_{1}^{\prime \prime}-\left(i \omega+\frac{1}{k}\right) u_{1}=-G r \theta_{1}-\left(i \omega+\frac{1}{k}\right), \\
& \theta_{1}^{\prime \prime}-i \omega \operatorname{Pr} \theta_{1}=0 .
\end{aligned}
$$


with the corresponding boundary conditions:

$$
\left.\begin{array}{ll}
y=0: & u_{0}=1, u_{1}=1, \theta_{0}=1, \theta_{1}=1 \\
y=1: & u_{0}=0, u_{1}=0, \theta_{0}=0, \theta_{1}=0,
\end{array}\right\}
$$

where primes denotes differentiation with respect to $y$. Solving these equations under the corresponding boundary conditions are

$$
\begin{aligned}
& \theta_{0}(y)=(1-y), \\
& \theta_{1}(y)=A e^{\lambda(1+i) y}+B e^{-\lambda(1+i) y}, \\
& u_{0}(y)=A_{1} e^{a_{1} y}+B_{1} e^{-a_{1} y}+G r k(1-y)+1, \\
& u_{1}(y)=A_{3} e^{\left(a_{2}+i a_{3}\right) y}+B_{2} e^{-\left(a_{2}+i a_{3}\right) y}-A A_{2} e^{\lambda(1+i) y}-B A_{2} e^{-\lambda(1+i)}+1,
\end{aligned}
$$

where:

$$
\begin{aligned}
& \lambda=\sqrt{\frac{\omega P r}{2}}, \quad A=-\frac{e^{-\lambda(1+i)}}{e^{\lambda(1+i)}-e^{-\lambda(1+i)}}, \quad B=\frac{e^{\lambda(1+i)}}{e^{\lambda(1+i)}-e^{-\lambda(1+i)}}, \\
& a_{1}=\frac{1}{\sqrt{k}}, \quad a_{2}=\frac{1}{\sqrt{2}}\left[\sqrt{\omega^{2}+\frac{1}{k^{2}}}+\frac{1}{k}\right]^{\frac{1}{2}}, \quad a_{3}=\frac{1}{\sqrt{2}}\left[\sqrt{\omega^{2}+\frac{1}{k^{2}}}-\frac{1}{k}\right]^{\frac{1}{2}}, \\
& A_{1}=-\left(\frac{1-G r k e^{-a_{1}}}{e^{a_{1}}-e^{-a_{1}}}\right), \quad B_{1}=\frac{\left(1-G r k e^{a_{1}}\right)}{e^{a_{1}}-e^{-a_{1}}}, \quad A_{2}=\frac{G r}{\lambda^{2}(1+i)^{2}-\left(i \omega+\frac{1}{k}\right)}, \\
& B_{2}=\frac{-\left[A A_{2}\left(e^{\left(a_{2}+i a_{3}\right)}-e^{\lambda(1+i)}\right)+B A_{2}\left(e^{\left(a_{2}+i a_{3}\right)}-e^{-\lambda(1+i)}\right)+1\right]}{\left[e^{-\left(a_{2}+i a_{3}\right)}-e^{\left(a_{2}+i a_{3}\right)}\right]} \\
& A_{3}=\frac{\left[A A_{2}\left(e^{-\left(a_{2}+i a_{3}\right)}-e^{\lambda(1+i)}\right)+B A_{2}\left(e^{-\left(a_{2}+i a_{3}\right)}-e^{-\lambda(1+i)}\right)+1\right]}{\left[e^{-\left(a_{2}+i a_{3}\right)}-e^{\left(a_{2}+i a_{3}\right)}\right]},
\end{aligned}
$$

\section{Result and discussions}

In order to point out the effect of permeability and convection on the velocity, when the moving plate is subjected to oscillating free-stream velocity and fluctuating wall temperature, the following discussions are set out. Numerical calculations are carried out for different values of $G r, P r, \omega$ and $k$. The values of Prandtl number are chosen as 0.71 and 7.0 approximately, which represent air and water respectively at $20^{\circ} \mathrm{C}$. The values of $G r$ and $k$ are selected arbitrarily.

\section{(a) Mean flow}

The mean flow velocity is given by equation (19). This velocity component is presented in Figure 1. It is observed from the figure that the mean velocity decreases with an increase in permeability parameter because the porous material offers resistance to the flow. 


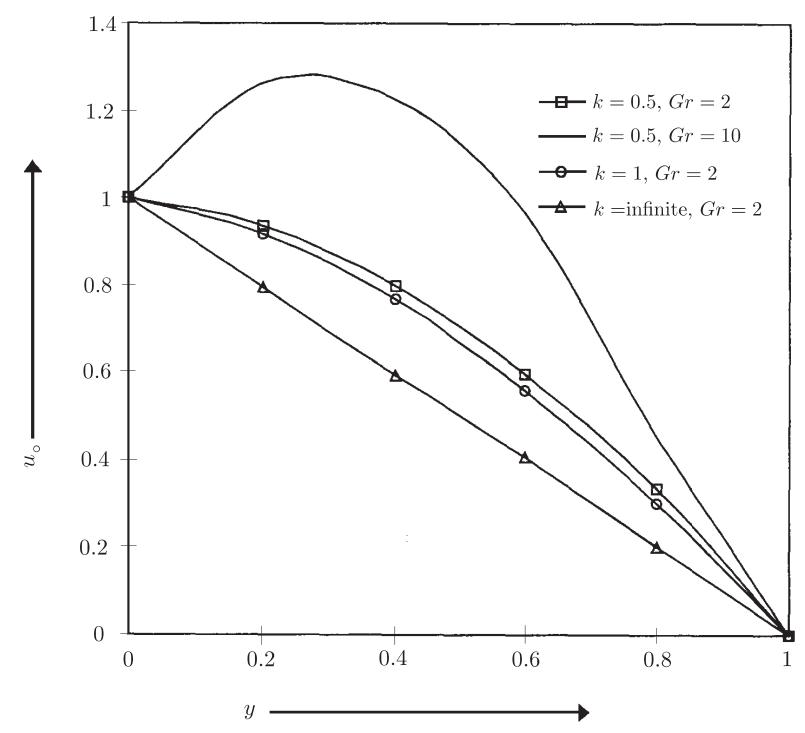

Figure 1. The mean flow velocity profiles.

Knowing the mean velocity field from the practical point of view, it is important to know that the effects of Grashoff number on mean-skin friction. It is given by:

$$
\tau^{*}=\mu\left(\frac{d u^{*}}{d y^{*}}\right)_{y^{*}=0}
$$

and in non-dimensional it is given by:

$$
\tau=\frac{\tau^{*} b}{\mu U_{0}}=\left(\frac{\partial u}{\partial y}\right)_{y=0}=\left(\frac{\partial u_{0}}{\partial y}\right)_{y=0}+\varepsilon\left(\frac{\partial u_{1}}{\partial y}\right)_{y=0} e^{i t} .
$$

Denoting the mean skin friction by

$$
\tau_{m}=\left(\frac{d u_{0}}{d y}\right)_{y=0}
$$

Substituting equation (19) in equation (22), we have

$$
\tau_{m}=A_{1} a_{1}-B_{1} a_{1}-G r k .
$$

The numerical values of $\tau_{m}$ are entered in Table 1 .

Table 1. The mean skin-friction $\left(\tau_{m}\right)$.

\begin{tabular}{|r|c|c|c|c|c|c|c|c|c|c|c|}
\hline$k$ & $\infty$ & 1 & 2 & 3 & 4 & 5 & 6 & 7 & 8 & 9 & 10 \\
\hline$G r=2$ & -1.00 & 1.77 & 3.72 & 5.70 & 7.69 & 9.69 & 11.68 & 13.68 & 15.68 & 17.68 & 19.68 \\
\hline$G r=5$ & -1.00 & 5.71 & 10.69 & 15.68 & 20.68 & 25.68 & 30.68 & 35.68 & 40.68 & 45.68 & 50.68 \\
\hline
\end{tabular}


It may be observed from this Table, the mean skin-friction increases with increase $k$ and $G r$ both. It is interesting to note that the mean skin-friction is more affected by increase in $G r$. This is interpreted physically as the presence of porous medium.

\section{(b) Unsteady flow}

The velocity and temperature fields as given by equations (17) to (20) respectively can be expressed in terms of fluctuating parts as follows

$$
\begin{aligned}
& u(y, t)=u_{0}(y)+\varepsilon e^{i t}\left(M_{r}+i M_{i}\right), \\
& \theta(y, t)=\theta_{0}(y)+\varepsilon e^{i t}\left(T_{r}+i T_{i}\right),
\end{aligned}
$$

where:

$$
M_{r}+i M_{i}=u_{1}(y) \text { and } T_{r}+i T_{i}=\theta_{1}(y) .
$$

We can now write expressions for transient velocity and transient temperature from (23) and (24) for $t=\pi / 2$, as follows

$$
\begin{aligned}
& u=(y, \pi / 2)=u_{0}(y)-\varepsilon M_{i}, \\
& \theta(y, \pi / 2)=\theta_{0}(y)-\varepsilon T_{i},
\end{aligned}
$$

The transient velocity profiles are shown in Figure 2. The graph reveals that the transient velocity increases with increasing $G r$ in both the situations $[P r=0.71$ (air) and $\operatorname{Pr}=7.0$ (water)], because of buoyancy force increasing in the upward direction. This figure further exhibits that the velocity decreases due to increase of permeability parameter $k$ in both the cases (air and water). Physically, this is true because the permeability of the porous medium exert retarding influence on the fluid motion. It is evident that for fixed values of $k$ and $G r$ the velocity increase as the frequency parameter $\omega$ increases for water $(\operatorname{Pr}=7.0)$, while reverse effect is observed for air $(\operatorname{Pr}=0.71)$. The values of transient velocity is more in air than in water because an increase in Prandtl number is due to an increase in the viscosity of the fluid which makes the fluid thick and hence decrease in the velocity. 


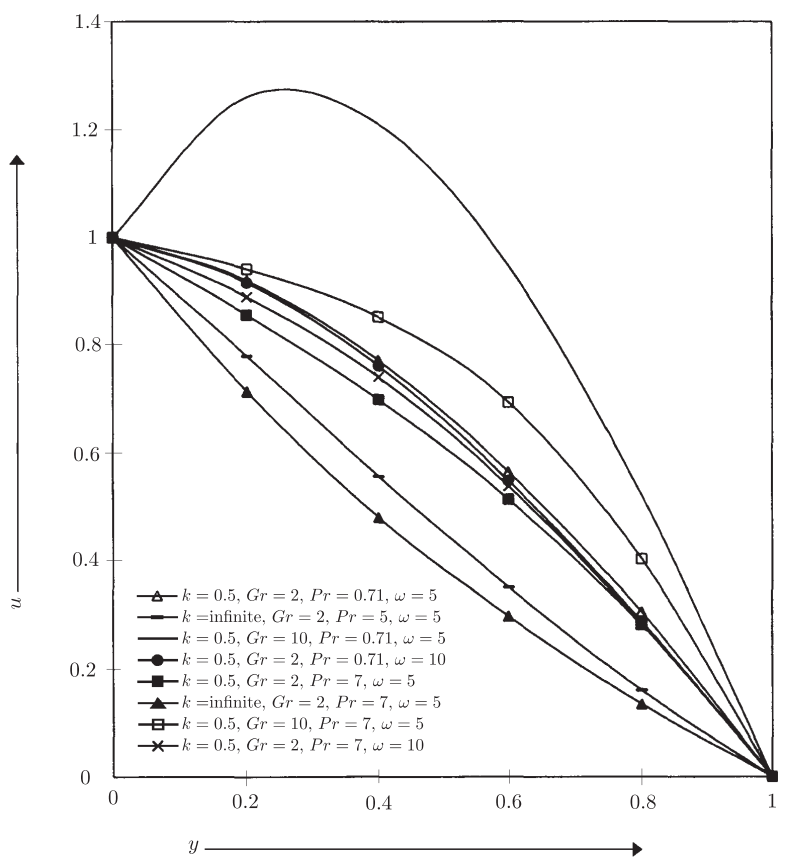

Figure 2. The transient velocity profiles for $t=\pi / 2$ and $\varepsilon=0.2$.

Figure 3. depicts the variation of temperature with distance from moving plate. It is observed that transient temperature increases with increasing $\omega$ for air $(\operatorname{Pr}=0.71)$, while reverse phenomena is observed for water $(\operatorname{Pr}=7.0)$. It is interesting to note that the values of transient temperature are greater near the moving plate for water while reverse effect is observed as we move towards the stationary plate. It is now proposed to study the behavior of amplitude and phase of skin-friction. From equations (20) and (21) we have

$$
\tau=\tau_{m}+\varepsilon e^{i t}\left[\left(a_{2}+i a_{3}\right) A_{3}-\left(a_{2}+i a_{3}\right) B_{2}-A A_{2} \lambda(1+i)+B A_{2} \lambda(1+i)\right] .
$$

We can express equation (27) in terms of the amplitude and phase of skin-friction as

$$
\tau=\tau_{m}+\varepsilon|M| \cos (t+\phi)
$$

where:

$$
\begin{aligned}
& M=M_{r}+i M_{i}=\text { coefficients of } \varepsilon e^{i t} \text { in equation (27) } \\
& |M|=\sqrt{M_{r}^{2}+M_{i}^{2}}, \quad \text { and } \tan \phi=M_{i} / M_{r} .
\end{aligned}
$$

The numerical values of $|M|$ are presented in Table 2 . It is observed from this table that $|M|$ increases with increasing $\omega$ for water while reverse effect is observed for air (for same values of $G r$ ). The increase in $G r$ leads to increase in amplitude of skin-friction. 




Figure 3. The transient temperature profiles for $\varepsilon=0.2$ and $t=\pi / 2$

Table 2. The values of $(|M|)$ for $k=0.5$.

\begin{tabular}{|l|l|l|l|l|}
\hline$\omega$ & $G r=2$ & $G r=1$ & $G r=2$ & $G r=1$ \\
& $P r=7$ & $P r=7$ & $P r=0.71$ & $\operatorname{Pr}=0.71$ \\
\hline 2 & 46.73 & 23.27 & 0.929 & 0.816 \\
4 & 58.56 & 28.97 & 0.722 & 0.658 \\
6 & 63.49 & 31.49 & 0.562 & 0.507 \\
8 & 71.34 & 35.69 & 0.449 & 0.351 \\
10 & 97.70 & 49.06 & 0.433 & 0.193 \\
\hline
\end{tabular}

The tangent of phase of skin-friction is reported in Figure 4. It is observed that phase of skin-friction increases with the increase in $G r$ in both the cases $(P r=0.71$ and $\operatorname{Pr}=7.0)$. The Figure 4 clearly shows that there is always a phase lead for small values of $k$ and a phase lag for large value of $k$ in case of air $(\operatorname{Pr}=0.71)$. The phase of skin-friction oscillates with increasing $\omega$ in case of water $(\operatorname{Pr}=7.0)$.

We now study the effect of $\omega$ on the rate of heat transfer. The rate of heat transfer in terms of the Nusselt number can be obtained as

$$
\begin{aligned}
& N u=-\frac{q_{\omega}^{*} b}{k\left(T_{n}^{*}-T_{s}^{*}\right)}=\left(\frac{\partial \theta}{\partial y}\right)_{y=0}=\left(\frac{\partial \theta_{0}}{\partial y}\right)_{y=0}+\varepsilon\left(\frac{\partial \theta_{1}}{\partial y}\right)_{y=0} e^{i t}, \\
& N_{u}=-1+\varepsilon e^{i t}[A \lambda(1+i)-B \lambda(1+i)] .
\end{aligned}
$$

We can express (30) in terms of amplitude and phase of heat transfer as

$$
N u=-1+\varepsilon|H| \cos (t+\psi),
$$


where

$$
\begin{aligned}
& H=H_{r}+i H_{i}=\text { coefficients of } \varepsilon e^{i t} \text { in expression (30) } \\
& |H|=\sqrt{H_{r}^{2}+H_{i}^{2}} \text { and } \tan \psi=H_{i} / H_{r} .
\end{aligned}
$$

The numerical values of the amplitude and phase of heat transfer are listed in Table 3.



Figure 4. The trangent of phase $\tan \phi$ of skin-friction.

Table 3. The amplitude and phase of heat transfer.

\begin{tabular}{|c|c|c|c|c|}
\hline \multirow{2}{*}{$\omega$} & \multicolumn{2}{|c|}{$|H|$} & \multicolumn{2}{c|}{$\tan \psi$} \\
\cline { 2 - 5 } & $\operatorname{Pr}=0.71$ & $\operatorname{Pr}=7.0$ & $\operatorname{Pr}=0.71$ & $\operatorname{Pr}=7.0$ \\
\hline 2 & 1.144 & 3.762 & 0.447 & 1.017 \\
4 & 1.474 & 5.293 & 0.773 & 0.997 \\
6 & 1.857 & 6.479 & 0.953 & 0.999 \\
8 & 2.228 & 7.483 & 1.032 & 1.000 \\
10 & 2.568 & 8.366 & 1.056 & 1.000 \\
\hline
\end{tabular}

We observe from the Table 3 that amplitude of heat transfer increases with increasing $\operatorname{Pr}$ and $\omega$ both. The values of amplitude are greater in case of water than in case of air. It is clear that there is always a phase lead for $\operatorname{Pr}=0.71$ (air), whenever the phase of heat transfer almost remains same for the case of water $(\operatorname{Pr}=7.0)$.

\section{Acknowledgement}

One of the authors Bhupendra Kumar Sharma is highly indebted to Council of Scientific and Industrial Research, New Delhi [INDIA] for award a S.R.F. 


\section{References}

[1] S. Ostrach, Laminar natural convection flow and heat transfer of fluids with and without heat sources in channels with constant wall temperature, NACA TN, (1952), 2863.

[2] S. Ostrach, New aspects of natural convection heat transfer, Trans. Am. Soc. Mec. Engrs. 75(1953), 1287-1290.

[3] V. M. Soundalgkar, Viscous dissipation effects on unsteady free convective flow past an infinite verical porous plate with constant suction, Int. J. Heat Mass Transfer, 15(1972), 1253-1261.

[4] A. Raptis, Unsteady free convection flow through a porous medium, Int. J. Engin. Sci. 21(1983), 345-348.

[5] A. Raptis and C. P. Peridikis, Oscillatory flow through a porous medium by the presence of free convective flow, Int. J. Engin. Sci. 23(1985), 51-55.

[6] D. A. Nield, Convection in a porous medium with inclined temperature gradient: An additionl results, Int. J. Heat Mass Transfer, 37(1994), 3021-3025.

[7] M. D. Kelleher and K. T. Yang, Heat transfer response of laminar free convection boundary layer along vertical heated plate to surface temperature oscillation, ZAMP 19(1968), 31-44.

[8] O. G. Martynenko, A. A. Berezovsky and Yu. A. Sokovishin, Laminar free convection from a vertical plate, Int. J. Heat Mass Transfer 27(1984), 869-881.

[9] S. D. Harris, D. B. Ingham and I. Pop, Free convection from a vertical plate in porous medium subjected to a sudden change in surface temperature, Int. Comm. Heat Mass Transfer 24(1997), 543-552.

[10] U. N. Das, R. K. Deka and V. M. Soundalgekar, Transient free convection flow past an infinite vertical plate with periodic temperature variation, J. Heat Transfer (ASME) 121(1999), 1091-1094.

[11] A. K. Singh, MHD free convective flow through a porous medium between two vertical parallel plates, Ind. J. Pure and Appl. Phys. 40(2002), 709-713.

Department of Mathematics, University of Rajasthan, Jaipur-302004, India.

E-mail: pawkumar20@yahoo.co.in

Department of Mathematics, University of Rajasthan, Jaipur-302004, India.

E-mail: bhupen_1402@yahoo.co.in

Department of Mathematics, University of Rajasthan, Jaipur-302004, India. 